\title{
EFFICIENT ALGORITHM FOR COMPUTING THE EULER-POINCARÉ CHARACTERISTIC OF A SEMI-ALGEBRAIC SET DEFINED BY FEW QUADRATIC INEQUALITIES
}

\author{
SAUGATA BASU
}

\begin{abstract}
We present an algorithm which takes as input a closed semialgebraic set, $S \subset \mathrm{R}^{k}$, defined by

$$
P_{1} \leq 0, \ldots, P_{\ell} \leq 0, P_{i} \in \mathrm{R}\left[X_{1}, \ldots, X_{k}\right], \operatorname{deg}\left(P_{i}\right) \leq 2,
$$

and computes the Euler-Poincaré characteristic of $S$. The complexity of the algorithm is $k^{O(\ell)}$.

Keywords. Semi-algebraic sets, Euler-Poincaré characteristic

Subject classification. 2000 Mathematics Subject Classification 14P10, 14P25
\end{abstract}

\section{Introduction}

Let $\mathrm{R}$ be a real closed field and let $S \subset \mathrm{R}^{k}$ be a basic semi-algebraic set defined by $P_{1} \leq 0, \ldots, P_{\ell} \leq 0$, with $P_{i} \in \mathrm{R}\left[X_{1}, \ldots, X_{k}\right], \operatorname{deg}\left(P_{i}\right) \leq 2,1 \leq i \leq \ell$. It is known [2, 3] that the sum of the Betti numbers of $S$ (and hence also its Euler-Poincaré characteristic) is bounded by $k^{O(\ell)}$. Notice that for fixed $\ell$, these bounds are polynomial in $k$. One can also check whether $S$ is non-empty, as well as compute a finite set of sample points meeting every connected component of $S$ in time $k^{O(\ell)}$ [2, 10]. However, no algorithm with similar complexity is known for computing any of the individual Betti numbers of $S$ (for instance, the number of connected components). The best known algorithm for computing all the Betti numbers of $S$ has complexity $k^{2^{O(\ell)}}[5]$.

Here, and elsewhere in this paper the Betti number, $b_{i}(S)$, is the dimension of the simplicial homology group, $H_{i}(S, \mathbb{Q})$, in case $S$ is closed and bounded. If $S$ is a closed but not necessarily bounded semi-algebraic set, $b_{i}(S)$ is the dimension of $H_{i}\left(S \cap \overline{B_{k}(0, r)}, \mathbb{Q}\right)$, for sufficiently large $r>0$ (here and in the rest of the paper, $B_{k}(0, r)$ denotes the open ball of radius $r$ in $R^{k}$ centered at 
the origin, and $\bar{X}$ denotes the closure of a semi-algebraic set $X)$. It is easy to see that $b_{i}(S)$ is well-defined and we denote by

$$
\chi(S)=\sum_{i=0}^{k}(-1)^{i} b_{i}(S)
$$

the Euler-Poincaré characteristic of $S$.

In this paper we describe an algorithm for computing the Euler-Poincaré characteristic of $S$, whose complexity is $k^{O(\ell)}$. Our algorithm relies on an efficient algorithm for computing the Euler-Poincaré characteristic of the realizations of all realizable sign conditions of a family of polynomials described in [6] and uses different techniques than those used in [2, 10].

The main result of this paper is the following.

Main Result: We present an algorithm (Algorithm 4.2 in Section 4) which given a set of $\ell$ polynomials, $\mathcal{P}=\left\{P_{1}, \ldots, P_{\ell}\right\} \subset \mathrm{R}\left[X_{1}, \ldots, X_{k}\right]$, with $\operatorname{deg}\left(P_{i}\right) \leq$ $2,1 \leq i \leq \ell$, computes the Euler-Poincaré characteristic, $\chi(S)$, where $S$ is the set defined by $P_{1} \leq 0, \ldots, P_{\ell} \leq 0$. The complexity of the algorithm is $k^{O(\ell)}$. If the coefficients of the polynomials in $\mathcal{P}$ are integers of bitsizes bounded by $\tau$, then the bitsizes of the integers appearing in the intermediate computations and the output are bounded by $\tau k^{O(\ell)}$.

The rest of the paper is organized as follows. In Section 2, we describe some mathematical and algorithmic results we will need for our algorithm. We also include a brief introduction to spectral sequences since they play a motivating role in the design of the main algorithm described in this paper. In Section 3 , we describe an algorithm for computing the Euler-Poincaré characteristic of a set defined by homogeneous quadratic inequalities. Finally, in Section 4 we describe our algorithm for the general (inhomogeneous) case.

For referring to well known results in real algebraic geometry we sometime use reference [7] as a useful source.

\section{Preliminaries}

In this section we describe some mathematical and algorithmic results we will require in the rest of the paper.

2.1. Definition of the Euler-Poincaré Characteristic. For the purposes of our algorithm, it will be useful to define Euler-Poincaré characteristic for locally closed semi-algebraic sets. We do this in terms of the Borel-Moore homology groups of such sets (defined below). This definition agrees with the definition of Euler-Poincaré characteristic stated earlier for closed and bounded 
semi-algebraic sets. They may be distinct for semi-algebraic sets which are closed but not bounded.

The simplicial homology groups of a pair of closed and bounded semialgebraic sets $T \subset S \subset \mathrm{R}^{k}$ are defined as follows. Such a pair of closed, bounded semi-algebraic sets can be triangulated [7] using a pair of simplicial complexes $(K, A)$, where $A$ is a sub-complex of $K$. The $p$-th simplicial homology group of the pair $(S, T), H_{p}(S, T)$, is $H_{p}(K, A)$. The dimension of $H_{p}(S, T)$ as a $\mathbb{Q}$-vector space is called the $p$-th Betti number of the pair $(S, T)$ and denoted $b_{p}(S, T)$. The Euler-Poincaré characteristic of the pair $(S, T)$ is

$$
\chi(S, T)=\sum_{i}(-1)^{i} b_{i}(S, T) .
$$

The $p$-th Borel-Moore homology group of $S \subset \mathrm{R}^{k}$, denoted $H_{p}^{B M}(S)$, is defined in terms of the homology groups of a pair of closed and bounded semialgebraic sets as follows. For $r>0$, let $S_{r}=S \cap B_{k}(0, r)$. Note that, for a locally closed semi-algebraic set $S$, both $\overline{S_{r}}$ and $\overline{S_{r}} \backslash S_{r}$ are closed and bounded and hence $H_{p}\left(\overline{S_{r}}, \overline{S_{r}} \backslash S_{r}\right)$ is well defined. Moreover, it is a consequence of Hardt's triviality theorem [11] that the homology group $H_{p}\left(\overline{S_{r}}, \overline{S_{r}} \backslash S_{r}\right)$ is invariant for all sufficiently large $r>0$. We define, $H_{p}^{B M}(S)=H_{p}\left(\overline{S_{r}}, \overline{S_{r}} \backslash S_{r}\right)$, for $r>0$ sufficiently large, and it follows from the remark above that it is well defined. The Borel-Moore homology groups are invariant under semi-algebraic homeomorphisms (see [8]. It also follows clearly from the definition that for a closed and bounded semi-algebraic set, the Borel-Moore homology groups coincide with the simplicial homology groups.

For a locally closed semi-algebraic set $S$, we define the Borel-Moore EulerPoincaré characteristic by,

$$
\chi^{B M}(S)=\sum_{i=0}^{k} b_{i}^{B M}(S),
$$

where $b_{i}^{B M}(S)$ denotes the dimension of $H_{i}^{B M}(S, \mathbb{Q})$. Note that if $S$ is closed and bounded, then $\chi^{B M}(S)=\chi(S)$.

The Borel-Moore Euler-Poincaré characteristic has the following additive property.

Proposition 2.1. Let $X, X_{1}$ and $X_{2}$ be locally closed semi-algebraic sets such that

$$
X_{1} \cup X_{2}=X, X_{1} \cap X_{2}=\emptyset .
$$

Then

$$
\chi^{B M}(X)=\chi^{B M}\left(X_{1}\right)+\chi^{B M}\left(X_{2}\right) .
$$


Proof. This is classical (see for example, Proposition 2.6 in [6] for a proof).

2.2. Sign Conditions and their realizations. A sign condition is an element of $\{0,1,-1\}$. We define

$$
\operatorname{sign}(x)= \begin{cases}0 & \text { if and only if } x=0 \\ 1 & \text { if and only if } x>0 \\ -1 & \text { if and only if } x<0\end{cases}
$$

Let $Z \subset \mathrm{R}^{k}$ be a locally closed semi-algebraic set and let $\mathcal{P}=\left\{P_{1}, \ldots, P_{s}\right\}$ be a finite subset of $\mathrm{R}\left[X_{1}, \ldots, X_{k}\right]$. A sign condition on $\mathcal{P}$ is an element of $\{0,1,-1\}^{\mathcal{P}}$.

The realization of the sign condition $\sigma$ on $Z$ is

$$
\mathcal{R}(\sigma, Z)=\left\{x \in Z \mid \bigwedge_{P \in \mathcal{P}} \operatorname{sign}(P(x))=\sigma(P)\right\},
$$

and its Euler-Poincaré characteristic is denoted $\chi^{B M}(\sigma, Z)$.

We denote by $\operatorname{Sign}(\mathcal{P}, Z)$ the list of $\sigma \in\{0,1,-1\}^{\mathcal{P}}$ such that $\mathcal{R}(\sigma, Z)$ is non-empty. We denote by $\chi^{B M}(\mathcal{P}, Z)$ the list of Euler-Poincaré characteristics $\chi^{B M}(\sigma, Z)=\chi^{B M}(\mathcal{R}(\sigma, Z))$ for $\sigma \in \operatorname{Sign}(\mathcal{P}, Z)$.

We will use the following algorithm for computing the list $\chi^{B M}(\mathcal{P}, Z)$ described in [6]. We describe here the input, output and complexity of the algorithm.

Algorithm 2.2. Euler-Poincaré Characteristic of Sign Conditions.

Input: an algebraic set $Z=\mathrm{Z}\left(Q, \mathrm{R}^{k}\right) \subset \mathrm{R}^{k}$ and a finite list $\mathcal{P}=P_{1}, \ldots, P_{s}$ of polynomials in $\mathrm{R}\left[X_{1}, \ldots, X_{k}\right]$.

Output: the list $\chi^{B M}(\mathcal{P}, Z)$.

Complexity: Let $k^{\prime}$ be the dimension of $Z, d$ a bound on the degree of $Q$ and the elements of $\mathcal{P}$ and $s=\#(\mathcal{P})$ ). The number of arithmetic operations is

$$
s^{k^{\prime}+1} O(d)^{k}+s^{k^{\prime}}\left(\left(k^{\prime} \log _{2}(s)+k \log _{2}(d)\right) d\right)^{O(k)} .
$$

The algorithm also involves the inversion matrices of size $s^{k^{\prime}} O(d)^{k}$ with integer coefficients.

If $\mathrm{D}=\mathbb{Z}$, and the bitsizes of the coefficients of the polynomials are bounded by $\tau$, then the bitsizes of the integers appearing in the intermediate computations and the output are bounded by $\tau\left(\left(k^{\prime} \log _{2}(s)+k \log _{2}(d)\right) d\right)^{O(k)}$. 
2.3. Infinitesimals. In our algorithms we will use infinitesimals in order to ensure that the set we are dealing with is bounded. To ensure this we will extend the ground field $\mathrm{R}$ to $\mathrm{R}\langle\varepsilon\rangle$, the real closed field of algebraic Puiseux series in $\varepsilon$ with coefficients in $\mathrm{R}$. The sign of a Puiseux series in $\mathrm{R}\langle\varepsilon\rangle$ agrees with the sign of the coefficient of the lowest degree term in $\varepsilon$. This induces a unique order on $\mathrm{R}\langle\varepsilon\rangle$ which makes $\varepsilon$ infinitesimal: $\varepsilon$ is positive and smaller than any positive element of $\mathrm{R}$.

If $\mathrm{R}^{\prime}$ is a real closed field containing $\mathrm{R}$, then given a semi-algebraic set $S$ in $\mathrm{R}^{k}$, we denote the extension of $S$ to $\mathrm{R}^{\prime}$ by $\operatorname{Ext}\left(S, \mathrm{R}^{\prime}\right) . \operatorname{Ext}\left(S, \mathrm{R}^{\prime}\right)$ is the semi-algebraic subset of $\mathrm{R}^{\prime k}$ defined by the same quantifier free formula that defines $S$. The set $\operatorname{Ext}\left(S, \mathrm{R}^{\prime}\right)$ is well defined (i.e. it only depends on the set $S$ and not on the quantifier free formula chosen to describe it). This is an easy consequence of the Tarski-Seidenberg transfer principle (see for example Section 2.4.1 in [7]).

2.4. Spectral Sequences. For the benefit of the readers we include a brief introduction to the theory of spectral sequences pointing to [9, 12] for more details.

A spectral sequence is a sequence of bigraded complexes $\left(E_{r}, d_{r}: E_{r}^{p, q} \rightarrow\right.$ $E_{r}^{p+r, q-r+1}$ ) such that the complex $E_{r+1}$ is obtained from $E_{r}$ by taking its homology with respect to $d_{r}$ (that is $E_{r+1}=H_{d_{r}}\left(E_{r}\right)$ ).

There are two spectral sequences, ' $E_{*}^{p, q},{ }^{\prime \prime} E_{*}^{p, q}$, (corresponding to taking rowwise or column-wise filtrations respectively) associated with a double complex $C^{\bullet \bullet \bullet}$, which will be important for us. Both of these converge to $H^{*}\left(\operatorname{Tot}^{\bullet}\left(C^{\bullet \bullet \bullet}\right)\right)$. This means that the homomorphisms $d_{r}$ are eventually zero, and hence the spectral sequences stabilize, and

$$
\bigoplus_{p+q=i}^{\prime} E_{\infty}^{p, q} \cong \bigoplus_{p+q=i}{ }^{\prime \prime} E_{\infty}^{p, q} \cong H^{i}\left(\operatorname{Tot}^{\bullet}\left(C^{\bullet, \bullet}\right)\right)
$$

for each $i \geq 0$.

The first terms of these are ' $E_{1}=H_{\delta}\left(C^{\bullet \bullet \bullet}\right),{ }^{\prime} E_{2}=H_{d} H_{\delta}\left(C^{\bullet \bullet \bullet}\right)$, and ${ }^{\prime \prime} E_{1}=$ $H_{d}\left(C^{\bullet \bullet \bullet}\right),{ }^{\prime \prime} E_{2}=H_{\delta} H_{d}\left(C^{\bullet \bullet \bullet}\right)$.

In particular, assuming that the complex $C^{\bullet \bullet \bullet}$ is bounded in both directions, we have that,

Proposition 2.3.

$$
\begin{aligned}
\sum_{i \geq 0}(-1)^{i} \operatorname{dim}\left(H^{i}\left(\operatorname{Tot}^{\bullet}\left(C^{\bullet, \bullet}\right)\right)\right) & =\sum_{p, q \geq 0}(-1)^{p+q} \operatorname{dim}\left({ }^{\prime} E_{2}^{p, q}\right) \\
& =\sum_{p, q \geq 0}(-1)^{p+q} \operatorname{dim}\left({ }^{\prime \prime} E_{2}^{p, q}\right)
\end{aligned}
$$




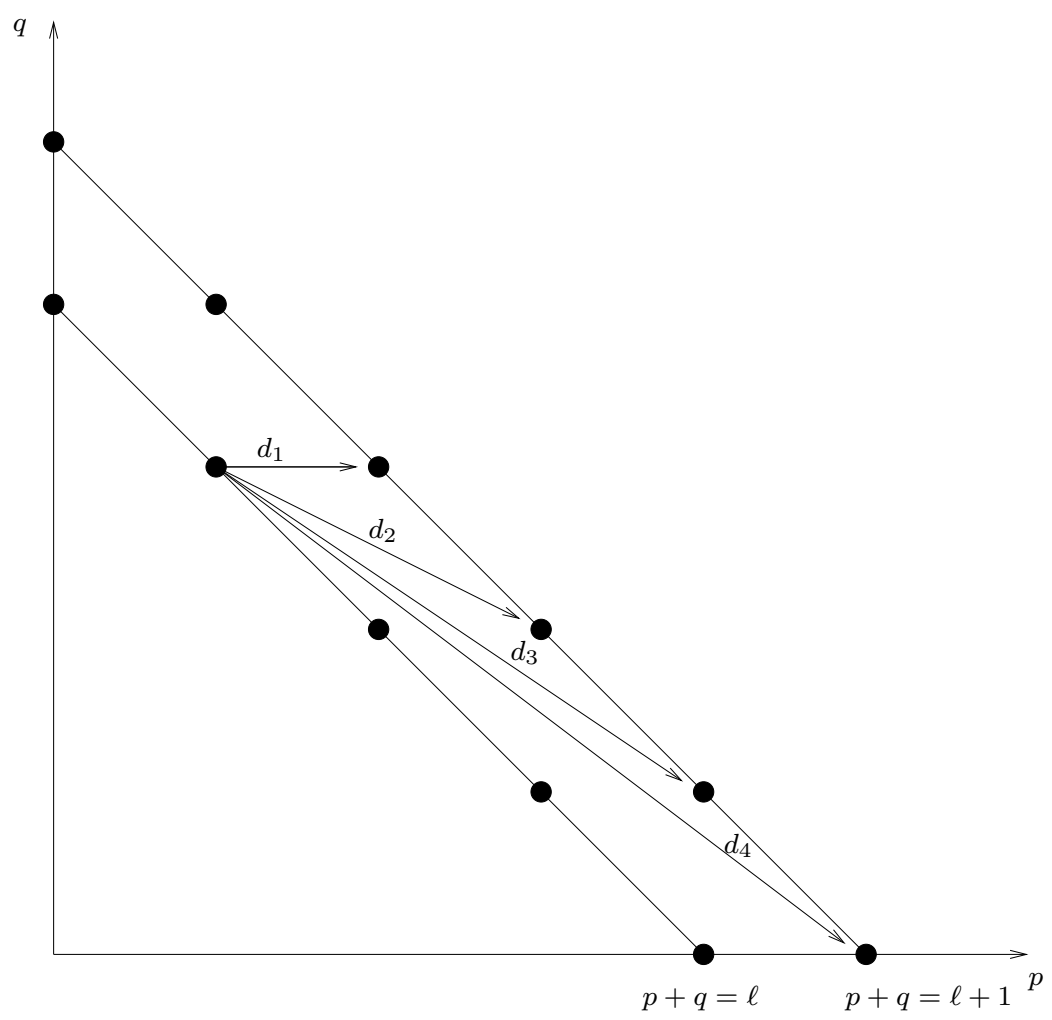

Figure 2.1: $d_{r}: E_{r}^{p, q} \rightarrow E_{r}^{p+r, q-r+1}$

2.5. Leray Spectral Sequence of a map. The Leray spectral sequence of a proper map,

$$
f: A \longrightarrow B
$$

is a classical tool in algebraic topology which relates the cohomology of the space $A$, with those of the space $B$ and of the fibers of the map $f$. Its most common use is in the theory of sheaf cohomology [9. We will need it in a very special situation where the sets $A$ and $B$ are compact semi-algebraic sets and $f$ a continuous semi-algebraic map. In this special situation it is possible to define the Leray spectral sequence in terms of triangulations, which we do below.

Consider a semi-algebraic continuous map, $f: A \longrightarrow B$, where $A$ and $B$ are compact semi-algebraic sets. Moreover, let $h: \Delta \longrightarrow B$ be a semi-algebraic triangulation of $B$, and let $\mathcal{H}(A)$ denote a cell-complex, such that $A$ is the union of the cells in $\mathcal{H}(A)$, and such that for any simplex $\sigma \in \Delta, A_{\sigma}=f^{-1}(\overline{h(\sigma)})$ is 
a subcomplex of $\mathcal{H}(A)$ ( where $\bar{X}$ denotes the topological closure of $X$ ).

Then, the Leray spectral sequence of $f$ is isomorphic to the spectral sequence (corresponding to the column-wise filtration) associated to the double complex $C^{\bullet, \bullet}$ defined as follows:

$$
C^{p, q}=\underset{\sigma \text { a } p \text {-simplex in } \Delta}{C^{q}\left(A_{\sigma}\right),}
$$

where $C^{q}\left(A_{\sigma}\right)$ denotes the vector space of $q$-co-chains of the complex $A_{\sigma}$. The horizontal and the vertical differentials are the obvious ones (see [9]). The spectral sequence associated to the double complex defined above converges to the co-homology of $A$.

\section{The basic homogeneous case}

Let $\mathcal{P}=\left\{P_{1}, \ldots, P_{\ell}\right\} \subset \mathrm{R}\left[X_{0}, X_{1}, \ldots, X_{k}\right]$ be a set of homogeneous quadratic polynomials, and let $S$ be the basic closed semi-algebraic set defined on the unit sphere $S^{k} \subset \mathrm{R}^{k+1}$ by the inequalities,

$$
P_{1} \leq 0, \ldots, P_{\ell} \leq 0 .
$$

We denote by $S_{i}$ the subset of $S^{k}$ defined by $P_{i} \leq 0$. Then, $S=\cap_{i=1}^{\ell} S_{i}$. For $J \subset\{1, \ldots, \ell\}$, we denote by $S^{J}=\cup_{j \in J} S_{j}$. The following equality is a consequence of the exactness of the Mayer-Vietoris sequence.

LEMMA 3.1.

$$
\chi(S)=\sum_{J \subset\{1, \ldots, \ell\}}(-1)^{\#(J)+1} \chi\left(S^{J}\right) .
$$

Proof. In case $\ell=2$, this is a direct consequence of the exactness of MayerVietoris sequence (see for example, [7, Corollary 6.28). The general case follows from an easy induction.

Thus, in order to compute $\chi(S)$ it suffices to compute $\chi\left(S^{J}\right)$ for each $J \subset$ $\{1, \ldots, \ell\}$.

3.1. Topology of sets defined by quadratic constraints. We first recall some facts about topology of sets defined by quadratic inequalities [1]. Let $P_{1}, \ldots, P_{s}$ be homogeneous quadratic polynomials in $\mathrm{R}\left[X_{0}, \ldots, X_{k}\right]$.

We denote by $P=\left(P_{1}, \ldots, P_{s}\right): \mathrm{R}^{k+1} \rightarrow \mathrm{R}^{s}$, the map defined by the polynomials $P_{1}, \ldots, P_{s}$. Let

$$
A=\bigcup_{1 \leq i \leq s}\left\{x \in S^{k} \mid P_{i}(x) \leq 0\right\} .
$$


Let

$$
\Omega=\left\{\omega \in R^{s}|| \omega \mid=1, \omega_{i} \leq 0,1 \leq i \leq s\right\}
$$

For $\omega \in \Omega$ we denote by $\omega P$ the quadratic form defined by

$$
\omega P=\sum_{i=1}^{s} \omega_{i} P_{i} .
$$

Let $B \subset \Omega \times S^{k}$ be the set defined by,

$$
B=\left\{(\omega, x) \mid \omega \in \Omega, x \in S^{k} \text { and } \omega P(x) \geq 0\right\} .
$$

We denote by $\phi_{1}: B \rightarrow \Omega$ and $\phi_{2}: B \rightarrow S^{k}$ the two projection maps.

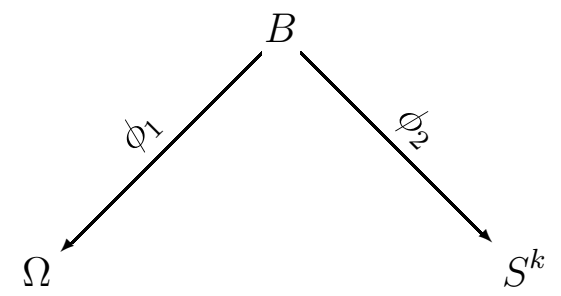

The following was proved by Agrachev [1]. With the notation developed above,

Proposition 3.2. The map $\phi_{2}$ gives a homotopy equivalence between $B$ and $\phi_{2}(B)=A$.

Proof. We first prove that $\phi_{2}(B)=A$. If $x \in A$, then there exists some $i, 1 \leq i \leq s$, such that $P_{i}(x) \leq 0$. Then for $\omega=\left(-\delta_{1 i}, \ldots,-\delta_{s i}\right)$ (where $\delta_{i j}=1$ if $i=j$, and 0 otherwise), we see that $(\omega, x) \in B$. Conversely, if $x \in \phi_{2}(B)$, then there exists $\omega=\left(\omega_{1}, \ldots, \omega_{s}\right) \in \Omega$ such that, $\sum_{i=1}^{s} \omega_{i} P_{i}(x) \geq 0$. Since, $\omega_{i} \leq 0,1 \leq i \leq s$, and not all $\omega_{i}=0$, this implies that $P_{i}(x) \leq 0$ for some $i, 1 \leq i \leq s$. This shows that $x \in A$.

For $x \in \phi_{2}(B)$, the fibre

$$
\phi_{2}^{-1}(x)=\{(\omega, x) \mid \omega \in \Omega \text { such that } \omega P(x) \geq 0\},
$$

can be identified with a non-empty subset of $\Omega$ defined by a single linear inequality. From convexity considerations, all such fibres can clearly be retracted to their center of mass continuously, proving the first half of the proposition. 
For any quadratic form $Q$, we will denote by index $(Q)$, the number of negative eigenvalues of the symmetric matrix of the corresponding bilinear form, that is of the matrix $M$ such that, $Q(x)=\langle M x, x\rangle$ for all $x \in R^{k+1}$. We will also denote by $\lambda_{i}(Q), 0 \leq i \leq k$, the eigenvalues of $Q$, in non-decreasing order, i.e.

$$
\lambda_{0}(Q) \leq \lambda_{1}(Q) \leq \cdots \leq \lambda_{k}(Q) .
$$

Given a quadratic map $P=\left(P_{1}, \ldots, P_{s}\right): \mathrm{R}^{k+1} \rightarrow \mathrm{R}^{s}$, and $0 \leq j \leq k$, we denote by

$$
\Omega_{j}=\left\{\omega \in \Omega \mid \lambda_{j}(\omega P) \geq 0\right\} .
$$

For notational convenience, $\Omega_{-1}$ will denote the empty set and $\Omega_{k+1}$ the whole space $\Omega$.

It is clear that the $\Omega_{j}$ 's induce a filtration of the space $\Omega$, i.e., $\Omega_{0} \subset \Omega_{1} \subset$ $\cdots \subset \Omega_{k+1}$.

Agrachev [1] showed that the Leray spectral sequence of the map $\phi_{1}$ (converging to the cohomology $\left.H^{*}(B) \cong H^{*}(A)\right)$, has as its $E_{2}$ terms,

$$
E_{2}^{p q}=H^{p}\left(\Omega_{k-q}, \Omega_{k-q-1}\right) .
$$

This follows from the fact that the fibre of the map $\phi_{1}$ over a point $\omega \in$ $\Omega_{j} \backslash \Omega_{j-1}$ has the homotopy type of a sphere of dimension $k-j$. To see this notice that for $\omega \in \Omega_{j} \backslash \Omega_{j-1}, \lambda_{0}(\omega P), \ldots, \lambda_{j-1}(\omega P)<0$. Moreover, letting $Y_{0}(\omega P), \ldots, Y_{k}(\omega P)$ be an orthonormal basis consisting of the eigenvectors of $\omega P$, we have that $\phi_{1}^{-1}(\omega)$ is the subset of $S^{k}$ defined by,

$$
\begin{gathered}
\sum_{i=0}^{k} \lambda_{i}(\omega P) Y_{i}(\omega P)^{2} \geq 0 \\
\sum_{i=0}^{k} Y_{i}(\omega P)^{2}=1
\end{gathered}
$$

Since, $\lambda_{i}(\omega P)<0,0 \leq i<j$, it follows that for $\omega \in \Omega_{j} \backslash \Omega_{j-1}, \phi_{1}^{-1}(\omega)$ is homotopy equivalent to the $(k-j)$-dimensional sphere defined by setting $Y_{0}(\omega P)=\cdots=Y_{j-1}(\omega P)=0$ on the sphere defined by $\sum_{i=0}^{k} Y_{i}(\omega P)^{2}=1$.

The following proposition relates the Euler-Poincaré characteristic of the set $A$ with those of $\Omega_{j} \backslash \Omega_{j-1}, 0 \leq j \leq k+1$.

Proposition 3.4.

$$
\chi(A)=\chi^{B M}(A)=\sum_{j=0}^{k+1} \chi^{B M}\left(\Omega_{j} \backslash \Omega_{j-1}\right)\left(1+(-1)^{(k-j)}\right) .
$$


Proof. Notice that the sets $\Omega_{j} \backslash \Omega_{j-1}$ are locally closed, and the fibre over a point $\omega \in \Omega_{j} \backslash \Omega_{j-1}$ is compact and has the homotopy type of a $(k-j)$ dimensional sphere. Now consider a sufficiently fine triangulation of $\Omega$, which respects the filtration $\Omega_{0} \subset \cdots \subset \Omega_{k+1}$, and such that over each simplex $\sigma$ of the triangulation lying in $\Omega_{j} \backslash \Omega_{j-1}, \phi_{1}^{-1}(\sigma)$ is homotopy equivalent to $\sigma \times S^{k-j}$. The Euler-Poincaré characteristic of a $(k-j)$-dimensional sphere, $S^{k-j}$, is equal to $1+(-1)^{(k-j)}$. The proposition now follows from the additivity property of the Borel-Moore Euler-Poincaré characteristic and Proposition 3.2.

Since Proposition 3.4 is central to the algorithm presented in this paper, we include a different proof below which uses the spectral sequence (3.3). First note that by Proposition 2.1.

$$
\chi^{B M}\left(\Omega_{j} \backslash \Omega_{j-1}\right)=\chi\left(\Omega_{j}\right)-\chi\left(\Omega_{j-1}\right)
$$

It follows from the convergence of the spectral sequence in (3.3) and Proposition 2.3 that,

$$
\begin{aligned}
\chi(A) & =\sum_{p+q=i}(-1)^{i} \operatorname{dim}\left(E_{2}^{p, q}\right) \\
& =\sum_{p+q=i}(-1)^{i} \operatorname{dim} H^{p}\left(\Omega_{k-q}, \Omega_{k-q-1}\right) \\
& =\sum_{0 \leq q \leq k+1} \sum_{0 \leq p \leq k}(-1)^{p+q} \operatorname{dim}\left(H^{p}\left(\Omega_{k-q}, \Omega_{k-q-1}\right)\right) \\
& =\sum_{0 \leq q \leq k+1}(-1)^{q} \sum_{0 \leq p \leq k}(-1)^{p} \operatorname{dim}\left(H^{p}\left(\Omega_{k-q}, \Omega_{k-q-1}\right)\right)
\end{aligned}
$$

Now, from the exact sequence of the pair $\left(\Omega_{k-q}, \Omega_{k-q-1}\right)$, namely,

$$
\cdots \rightarrow H^{i-1}\left(\Omega_{k-q}\right) \rightarrow H^{i-1}\left(\Omega_{k-q-1}\right) \rightarrow H^{i}\left(\Omega_{k-q}, \Omega_{k-q-1}\right) \rightarrow H^{i}\left(\Omega_{k-q}\right) \rightarrow \cdots
$$

we get that,

$$
\sum_{i \geq 0}(-1)^{i}\left(\operatorname{dim}\left(H^{i}\left(\Omega_{k-q-1}\right)\right)-\operatorname{dim}\left(H^{i}\left(\Omega_{k-q}\right)\right)+\operatorname{dim}\left(H^{i}\left(\Omega_{k-q}, \Omega_{k-q-1}\right)\right)=0\right.
$$

which yields

$$
\sum_{0 \leq p \leq k}(-1)^{p} \operatorname{dim}\left(H^{p}\left(\Omega_{k-q}, \Omega_{k-q-1}\right)\right)=\chi\left(\Omega_{k-q}\right)-\chi\left(\Omega_{k-q-1}\right) .
$$


Thus, the previous sum

$$
\begin{aligned}
& =\sum_{0 \leq q \leq k+1}(-1)^{q}\left(\chi\left(\Omega_{k-q}\right)-\chi\left(\Omega_{k-q-1}\right)\right) \\
& =\sum_{0 \leq j \leq k+1}(-1)^{k+1-j}\left(\chi\left(\Omega_{j-1}\right)-\chi\left(\Omega_{j-2}\right)\right) \\
& =\sum_{0 \leq j \leq k+1}(-1)^{k+1-j} \chi\left(\Omega_{j-1}\right)-\sum_{0 \leq j \leq k+1}(-1)^{k+1-j} \chi\left(\Omega_{j-2}\right) \\
& =\sum_{0 \leq j \leq k}(-1)^{k-j} \chi\left(\Omega_{j}\right)-\sum_{0 \leq j \leq k-1}(-1)^{k+1-j} \chi\left(\Omega_{j}\right) \\
& =\sum_{0 \leq j \leq k}(-1)^{k-j} \chi\left(\Omega_{j}\right)+\sum_{0 \leq j \leq k-1}(-1)^{k-j} \chi\left(\Omega_{j}\right) \\
& =\chi\left(\Omega_{k}\right)-2 \chi\left(\Omega_{k-1}\right)+2 \chi\left(\Omega_{k-2}\right)+\cdots+(-1)^{k} 2 \chi\left(\Omega_{0}\right) \\
& =\sum_{0 \leq j \leq k+1}\left(\chi\left(\Omega_{j}\right)-\chi\left(\Omega_{j-1}\right)\right)\left(1+(-1)^{k-j}\right) \\
& =\sum_{0 \leq j \leq k+1} \chi^{B M}\left(\Omega_{j} \backslash \Omega_{j-1}\right)\left(1+(-1)^{k-j}\right) .
\end{aligned}
$$

Let $Z=\left(Z_{1}, \ldots, Z_{s}\right)$ be variables and let $M(Z)$ be the symmetric matrix corresponding to the quadratic form $Z \cdot P=Z_{1} P_{1}+\cdots+Z_{s} P_{s}$. The entries of $M(Z)$ depend linearly on $Z$. Let

$$
F(Z, T)=\operatorname{det}\left(M(Z)+T \cdot I_{k+1}\right)=T^{k+1}+C_{k} T^{k}+\cdots+C_{0},
$$

where each $C_{i} \in \mathrm{R}\left[Z_{1}, \ldots, Z_{s}\right]$ is a polynomial of degree at most $k+1$.

It follows from the well known Descarte's rule of signs (see for example, Remark 2.42 in [7]) that for any $z \in \Omega$, index $(z P)$ is equal to the number of sign variations in the sequence $C_{0}(z), \ldots, C_{k}(z),+1$. Thus, the signs of the polynomials $C_{0}, \ldots, C_{k}$ determine the index of $z P$. For $\sigma \in\{0,+1,-1\}^{\mathcal{C}}$ a sign condition on the family $\mathcal{C}=\left\{C_{0}, \ldots, \mathrm{C}_{k}\right\}$, let $n(\sigma)$ denote the number of sign variations in the sequence, $\sigma\left(C_{0}\right), \ldots, \sigma\left(C_{k}\right),+1$. Let $\operatorname{Sign}(\mathcal{C}, \Omega)$ be the set of sign conditions realized by the family $\mathcal{C}$ on $\Omega$. The following proposition is an immediate consequence of Proposition 3.4 and the additivity of the EulerPoincaré characteristic.

Proposition 3.5.

$$
\chi(A)=\chi^{B M}(A)=\sum_{\sigma \in \operatorname{Sign}(\mathcal{C}, \Omega)} \chi^{B M}(\mathcal{R}(\sigma, \Omega)) \cdot\left(1+(-1)^{(k-n(\sigma))}\right) .
$$

Before proceeding further we discuss a small example.

ExAmple 3.6. Let $\ell=2, k=2$ and $P=\left(P_{1}, P_{2}\right): \mathrm{R}^{3} \rightarrow \mathrm{R}^{2}$ be the quadratic map with,

$$
\begin{aligned}
& P_{1}=X_{0}^{2}+X_{1}^{2}-X_{2}^{2}, \\
& P_{2}=X_{0}^{2}-X_{1}^{2}-X_{2}^{2} .
\end{aligned}
$$


In this example,

$$
\Omega=\left\{\left(\omega_{1}, \omega_{2}\right) \mid \omega_{1}^{2}+\omega_{2}^{2}=1, \omega_{1}, \omega_{2} \leq 0\right\}
$$

consists of the arc of the unit circle in the third quadrant.

Also,

$$
\begin{aligned}
A & =\left\{x \in S^{2} \mid P_{1}(x) \leq 0 \vee P_{2}(x) \leq 0\right\} \\
& =\left\{\left(x_{0}, x_{1}, x_{2}\right) \in \mathrm{R}^{3} \mid x_{0}^{2}+x_{1}^{2}+x_{2}^{2}=1, x_{1}^{2}+x_{2}^{2} \geq 1 / 2\right\} .
\end{aligned}
$$

The set $A$ in this example clearly has the homotopy type of a circle, and hence,

$$
\chi(A)=0 .
$$

Now, for $\omega=\left(\omega_{1}, \omega_{2}\right) \in \Omega$,

$$
\begin{aligned}
\omega P & =\omega_{1} P_{1}+\omega_{2} P_{2} \\
& =\left(\omega_{1}+\omega_{2}\right) X_{0}^{2}+\left(\omega_{1}-\omega_{2}\right) X_{1}^{2}-\left(\omega_{1}+\omega_{2}\right) X_{2}^{2} .
\end{aligned}
$$

Following notations introduced above,

$$
\begin{aligned}
& F\left(Z_{1}, Z_{2}, T\right)=\left(Z_{1}+Z_{2}+T\right)\left(Z_{1}-Z_{2}+T\right)\left(-Z_{1}-Z_{2}+T\right) \\
& =T^{3}+\left(Z_{1}-Z_{2}\right) T^{2}-\left(Z_{1}+Z_{2}\right)^{2} T+\left(Z_{1}+Z_{2}\right)^{2}\left(Z_{2}-Z_{1}\right) .
\end{aligned}
$$

Thus, in this example,

$$
\begin{gathered}
C_{0}\left(Z_{1}, Z_{2}\right)=\left(Z_{1}+Z_{2}\right)^{2}\left(Z_{2}-Z_{1}\right), \\
C_{1}\left(Z_{1}, Z_{2}\right)=-\left(Z_{1}+Z_{2}\right)^{2} \\
C_{2}\left(Z_{1}, Z_{2}\right)=Z_{1}-Z_{2} .
\end{gathered}
$$

There are three realizable sign conditions on $\mathcal{C}=\left\{C_{0}, C_{1}, C_{2},+1\right\}$ on $\Omega$. They are,

$$
\begin{gathered}
\sigma_{1}=(-,-,+,+), \\
\sigma_{2}=(0,-, 0,+), \\
\sigma_{3}=(+,-,-,+) .
\end{gathered}
$$

We have,

$$
\begin{aligned}
& n\left(\sigma_{1}\right)=1, \\
& n\left(\sigma_{2}\right)=1,
\end{aligned}
$$




$$
n\left(\sigma_{3}\right)=2 .
$$

The realizations $\mathcal{R}\left(\sigma_{1}, \Omega\right)$ and $\mathcal{R}\left(\sigma_{3}, \Omega\right)$ are each homeomorphic to $[0,1)$ while $\mathcal{R}\left(\sigma_{2}, \Omega\right)$ is a point. Thus,

$$
\chi^{B M}\left(\sigma_{1}, \Omega\right)=\chi^{B M}\left(\sigma_{3}, \Omega\right)=0,
$$

while

$$
\chi^{B M}\left(\sigma_{2}, \Omega\right)=1
$$

Finally, we have

$$
\begin{aligned}
\sum_{j=1}^{3} \chi^{B M}\left(\sigma_{j}, \Omega\right)\left(1-(-1)^{2-n\left(\sigma_{j}\right)}\right)= & 0\left(1+(-1)^{1}\right)+1\left(1+(-1)^{1}\right) \\
& +0\left(1+(-1)^{2}\right) \\
= & 0,
\end{aligned}
$$

which agrees with (3.7),

We are now in a position to describe an algorithm for computing the Euler-Poincaré characteristic of a union of sets, each defined by a homogeneous quadratic inequality. In the algorithm we will use the following notation. Given two finite families of polynomials, $\mathcal{P} \subset \mathcal{P}^{\prime}$, and $\sigma \in\{0,+1,-1\}^{\mathcal{P}}, \sigma^{\prime} \in$ $\{0,+1,-1\}^{\mathcal{P}^{\prime}}$, we say that $\sigma \prec \sigma^{\prime}$ iff for all $P \in \mathcal{P}, \sigma(P)=\sigma^{\prime}(P)$.

Algorithm 3.8. Euler-Poincaré characteristic of a union.

Input: A set of quadratic forms $\left\{P_{1}, \ldots, P_{s}\right\} \subset \mathrm{R}\left[X_{0}, \ldots, X_{k}\right]$.

Output: $\chi(A)$, where $A$ is the set defined on the unit sphere $S^{k} \subset \mathrm{R}^{k+1}$ by the formula

$$
P_{1} \leq 0 \vee \cdots \vee P_{\ell} \leq 0 .
$$

1. Let $P=\left(P_{1}, \ldots, P_{s}\right)$. Let $Z=\left(Z_{1}, \ldots, Z_{s}\right)$ be variables and let $M(Z)$ be the symmetric matrix corresponding to the quadratic form $Z \cdot P$. Compute the polynomials $C_{i} \in \mathrm{R}\left[Z_{1}, \ldots, Z_{s}\right]$ by computing the following determinant.

$$
\operatorname{det}\left(M(Z)+T \cdot I_{k}\right)=T^{k+1}+C_{k} T^{k}+\cdots+C_{0} .
$$

2. Compute $\chi^{B M}(\mathcal{C}, \Omega)$ as follows. Call Algorithm 2.2 with input $\mathcal{C}^{\prime}=\mathcal{C} \cup$ $\left\{Z_{1}, \ldots, Z_{s}\right\}$ and $Q=Z_{1}^{2}+\cdots+Z_{s}^{2}-1$. The output is the list

$$
\chi^{B M}\left(\mathcal{C}^{\prime}, \mathrm{Z}\left(Q, \mathrm{R}^{k}\right)\right) \text {. }
$$

For each $\sigma \in\{0,+1,-1\}^{\mathcal{C}}$, such that there exists $\sigma^{\prime} \in \operatorname{Sign}\left(\mathcal{C}^{\prime}, \mathrm{Z}\left(Q, \mathrm{R}^{k}\right)\right)$ with $\sigma \prec \sigma^{\prime}$ and $\sigma^{\prime}\left(Z_{j}\right) \in\{0,-1\}$ for $1 \leq j \leq s$, compute

$$
\chi^{B M}(\sigma, \Omega)=\sum_{\sigma^{\prime}, \sigma \prec \sigma^{\prime}, \sigma^{\prime}\left(Z_{j}\right) \in\{0,-1\}, 1 \leq j \leq s} \chi^{B M}\left(\sigma^{\prime}, \mathrm{Z}\left(Q, \mathrm{R}^{k}\right)\right) .
$$


3. Output

$$
\chi(A)=\sum_{\sigma \in \operatorname{Sign}(\mathcal{C}, \Omega)} \chi^{B M}(\mathcal{R}(\sigma, \Omega)) \cdot\left(1+(-1)^{(k-n(\sigma))}\right) .
$$

Proof of Correctness: The correctness of the algorithm is a consequence of Proposition 3.5 and the correctness of Algorithm 2.2.

COMPlEXITY AnAlysis: The complexity of the algorithm is $k^{O(s)}$ using the complexity of Algorithm 2.2.

We are now in a position to describe the algorithm for computing the EulerPoincaré characteristic in the basic, homogeneous case.

Algorithm 3.9. The basic homogeneous case.

Input: A set of quadratic forms $\left\{P_{1}, \ldots, P_{\ell}\right\} \subset \mathrm{R}\left[X_{0}, \ldots, X_{k}\right]$.

Output: $\chi(S)$, where $S$ is the set defined on the unit sphere $S^{k} \subset \mathrm{R}^{k+1}$ by the inequalities,

$$
P_{1} \leq 0, \ldots, P_{\ell} \leq 0
$$

1. For each subset $J \subset\{1, \ldots, \ell\}$ do the following.

2. Compute using Algorithm 3.8 $\chi\left(S^{J}\right)$.

3. Output

$$
\chi(S)=\sum_{J \subset\{1, \ldots, \ell\}}(-1)^{\#(J)+1} \chi\left(S^{J}\right) .
$$

Proof of Correctness: The correctness of the algorithm is a consequence of Lemma Lemma 3.1 and the correctness of Algorithm 3.8.

Complexity Analysis: There are $2^{\ell}$ calls to Algorithm 3.8 . Using the complexity analysis of Algorithm 3.8, the complexity of the algorithm is bounded by $k^{O(\ell)}$.

\section{The General Case}

Let $\mathcal{P}=\left\{P_{1}, \ldots, P_{\ell}\right\} \subset \mathrm{R}\left[X_{1}, \ldots, X_{k}\right]$ with $\operatorname{deg}\left(P_{i}\right) \leq 2,1 \leq i \leq \ell$, and let $S \subset \mathrm{R}^{k}$ be the basic semi-algebraic set defined by $P_{1} \leq 0, \ldots, P_{\ell} \leq 0$. Let $0<\varepsilon$ be an infinitesimal, and let

$$
P_{\ell+1}=\varepsilon \sum_{j=1}^{k} X_{j}^{2}-1
$$


Let $S^{\prime} \subset \mathrm{R}\langle\varepsilon\rangle^{k}$ be the set defined by $P_{1} \leq 0, \ldots, P_{\ell+1} \leq 0$.

Denoting by $P_{i}^{h}$ the homogenization of $P_{i}$, and $S^{h} \subset S^{k}$ the set defined by, $P_{1}^{h} \leq 0, \ldots, P_{\ell}^{h} \leq 0, P_{\ell+1}^{h} \leq 0$, on the unit sphere in $\mathrm{R}\langle\varepsilon\rangle^{k+1}$ we have,

Proposition 4.1. For $0 \leq i \leq k, \chi(S)=\chi\left(S^{\prime}\right)=\frac{1}{2} \chi\left(S^{h}\right)$.

Proof. Using the well known conic structure at infinity of semi-algebraic sets (see for example Proposition 5.50, 7]) we have that for all sufficiently large $r>0, S \cap \overline{B_{k}(0, r)}$ is a semi-algebraic deformation retract of $S$. Since $\varepsilon$ is an infinitesimal, it follows that $S^{\prime}=\operatorname{Ext}(S, \mathrm{R}\langle\varepsilon\rangle) \cap \overline{B_{k}\left(0, \frac{1}{\varepsilon}\right)}$ is a semi-algebraic deformation retract of $\operatorname{Ext}(S, \mathrm{R}\langle\varepsilon\rangle)$. This implies that $\chi(S)=\chi\left(S^{\prime}\right)$.

To prove the second equality, first observe that $S^{\prime}$ is bounded, and $S^{h}$ is the projection from the origin of the set $1 \times S^{\prime} \subset 1 \times \mathrm{R}\langle\varepsilon\rangle^{k}$ onto the unit sphere in $\mathrm{R}^{k+1}$. Since, $S^{\prime}$ is bounded, the projection does not intersect the equator and consists of two disjoint copies in the upper and lower hemispheres, and each copy is homeomorphic to $S^{\prime}$.

Algorithm 4.2. The general case.

Input: A family of polynomials $\mathcal{P}=\left\{P_{1}, \ldots, P_{\ell}\right\} \subset \mathrm{R}\left[X_{1} \ldots, X_{k}\right]$, with $\operatorname{deg}\left(P_{i}\right) \leq$ 2 .

Output: $\chi(S)$, where $S$ is the set defined by

$$
S=\bigcap_{P \in \mathcal{P}}\left\{x \in \mathrm{R}^{k} \mid P(x) \leq 0\right\} .
$$

1. Replace the family $\mathcal{P}$ by the family, $\mathcal{P}^{h}=\left\{P_{1}^{h}, \ldots, P_{\ell}^{h}, P_{\ell+1}^{h}\right\}$.

2. Using Algorithm 3.9 compute $\chi\left(S^{h}\right)$.

3. Output $\chi(S)=\frac{1}{2} \chi\left(S^{h}\right)$.

Proof of Correctness: The correctness of Algorithm 4.2 is a consequence of Proposition 4.1 and the correctness of Algorithm 3.9.

Complexity Analysis: The complexity of the algorithm is clearly $k^{O(\ell)}$ from complexity analysis of Algorithm 3.9.

REMARK 4.3. In this paper we have described an algorithm for computing the Euler-Poincaré characteristic of a basic closed semi-algebraic set defined by a constant number of quadratic inequalities $P \leq 0, P \in \mathcal{P}$. It is straightforward to extend the algorithm to the case of semi-algebraic sets defined by Boolean formulas without negations, whose atoms are of the form $P \geq 0$ or $P \leq 0$ for 
$P \in \mathcal{P}$, using the technique used in 4] for reducing the problem of computing Euler-Poincaré characteristic of such sets, to the basic closed case. This reduction works perfectly well even in the quadratic situation and does not worsen the complexity.

\section{Acknowledgements}

The author was supported in part by an NSF Career Award 0133597 and a Sloan Foundation Fellowship.

\section{References}

[1] A.A. Agrachev, Topology of quadratic maps and Hessians of smooth maps, Algebra, Topology, Geometry, Vol 26 (Russian),85-124, 162, Itogi Nauki i Tekhniki, Akad. Nauk SSSR, Vsesoyuz. Inst. Nauchn.i Tekhn. Inform., Moscow, 1988. Translated in J. Soviet Mathematics. 49 (1990), no. 3, 990-1013.

[2] A.I. Barvinok, Feasability Testing for Systems of Real Quadratic Equations, Discrete and Computational Geometry, 10:1-13 (1993).

[3] S. BAsu, On different bounds on different Betti numbers, Discrete and Computational Geometry, Vol 30, No. 1 (2003).

[4] S. BASU, On Bounding the Betti Numbers and Computing the Euler Characteristics of Semi-algebraic Sets, Discrete and Computational Geometry, 22 1-18 (1999).

[5] S. BAsu, Polynomial time algorithm for computing the top Betti numbers of Semi-algebraic sets defined by quadratic inequalities, preprint.

[6] S. Basu, R. Pollack, M.-F. Roy, Computing the Euler-Poincaré characteristic of sign conditions, Computational Complexity, 14:53-71 (2005).

[7] S. Basu, R. Pollack, M.-F. Roy, Algorithms in Real Algebraic Geometry, Springer-Verlag, 2003.

[8] J. Bochnak, M. Coste, M.-F. Roy, Géométrie algébrique réelle, Springer-Verlag (1987). Real algebraic geometry, Springer-Verlag (1998).

[9] G. E. Bredon, Sheaf Theory, Springer-Verlag (1996). 
[10] D. Grigor'ev, D.V. Pasechnik, Polynomial time computing over quadratic maps I. Sampling in real algberaic sets, Computational Complexity, 14:20-52 (2005).

[11] R. M. HARdT, Semi-algebraic Local Triviality in Semi-algebraic Mappings, Am. J. Math. 102, 291-302 (1980).

[12] J. McCleary A User's Guide to Spectral Sequences, Second Edition Cambridge Studies in Advanced Mathematics, 2001.

Manuscript received March 21, 2005.

SAUGATA BAsU

School of Mathematics, Georgia Institute of Technology, Atlanta, GA 30332, U.S.A.

saugata@math.gatech.edu 